\title{
Alexandre Papas, Mystiques et vagabonds en islam : portraits de trois soufis qalandar
}

Paris, Éditions du Cerf, coll. « Patrimoines - Visages de l'islam », 2010, $338 \mathrm{p}$.

\section{Stéphane A. Dudoignon}

\section{CpenEdition}

Journals

Édition électronique

URL : http://journals.openedition.org/assr/22861

DOI : $10.4000 /$ assr.22861

ISSN : $1777-5825$

Éditeur

Éditions de l'EHESS

Édition imprimée

Date de publication : 31 décembre 2011

ISBN : 9782713223273

ISSN : 0335-5985

\section{Référence électronique}

Stéphane A. Dudoignon, « Alexandre Papas, Mystiques et vagabonds en islam : portraits de trois soufis qalandar », Archives de sciences sociales des religions [En ligne], 156 | octobre-décembre 2011 mis en ligne le 29 novembre 2013, consulté le 21 septembre 2020. URL : http:// journals.openedition.org/assr/22861 ; DOI : https://doi.org/10.4000/assr.22861

Ce document a été généré automatiquement le 21 septembre 2020.

(c) Archives de sciences sociales des religions 


\section{Alexandre Papas, Mystiques et vagabonds en islam : portraits de trois soufis qalandar}

Paris, Éditions du Cerf, coll. « Patrimoines - Visages de l'islam », 2010, $338 \mathrm{p}$.

\section{Stéphane A. Dudoignon}

\section{RÉFÉRENCE}

Alexandre PAPAS, Mystiques et vagabonds en islam : portraits de trois soufis qalandar, Paris, Éditions du Cerf, coll. « Patrimoines - Visages de l'islam », 2010, 338 p.

156-88

1 Déjà auteur d'un ouvrage de référence, bâti sur une thèse de doctorat, Soufisme et politique entre Chine, Tibet et Turkestan (Paris, Jean Maisonneuve, 2005), ainsi que d'un très récent récit de voyage au carrefour historique du Qinghai (Voyage au pays des Salars (Tibet oriental, début $d u$ XXI ${ }^{e}$ siècle), Paris, Cartouche, 2011), Alexandre Papas nous offre une nouvelle variation, littéraire cette fois, d'histoire culturelle et religieuse de l'Asie Centrale pré-moderne avec ce recueil de trois essais, agrémentés de nombreux passages de poésie gnostique en traduction, sur trois figures majeures du soufisme de langue turcique et de tradition qalandar du Turkestan oriental entre la fin du xviI et le milieu du XVIII ${ }^{\mathrm{e}}$ siècle.

2 Qu'est-ce que la Qalandariyya ? Si l'ouvrage ne prétend pas retracer l'histoire de cette voie gnostique particulière de l'islam, très répandue jusqu'au début $d u x^{e}$ siècle dans les mondes iranien, turc et indien, il offre, à travers l'itinéraire de trois soufis errants et l'étude de leur œuvre et de leur hagiographie respective, une réflexion sur ce que le vagabondage volontaire, la pauvreté et une pratique systématique de la provocation révèlent de la société centrasiatique à la veille de l'époque moderne, et sur ce que ces "pauvres en Dieu» peuvent nous apprendre d'une période historique médiocrement 
documentée. Inversement, cet effort de documentation jette un éclairage neuf sur l'histoire de la Qalandariyya elle-même, notamment dans ses relations avec l'autre grande voie gnostique musulmane de la même période en Asie Centrale, la Naqšbandiyya mieux connue des historiens, sinon du grand public.

Des trois poètes gnostiques dont le présent ouvrage offre le portrait, le premier, Bābā Rahīm Mašrab (1640-1711), natif de Namangan dans l'actuel Ferghana ouzbek, est sans doute le mieux connu (y compris du public francophone, grâce à un premier essai de traduction malheureusement un peu bâclé : Le vagabond flamboyant : anecdotes et poèmes soufis, traduction de J.-P. Balpe et H. Ismailov, Gallimard coll. "Connaissance de l'Orient », 1993), sans omettre Les Envolées de Mašrab [Polëty Mašraba], drame du metteur en scène ouzbékistanais Marc Weil (1952-2007) régulièrement représenté depuis 2006 au Théâtre Ilhom de Tachkent - et dont une traduction française, inédite, a été réalisée). Mašrab demeure cependant le plus mystérieux des trois personnages traités ici. Parmi l'abondante postérité apocryphe du poète vagabond, l'auteur opère un tri drastique - acte familier des historiens du soufisme dans le monde turc- en concentrant son récit sur la source la plus fiable le concernant, à savoir, paradoxalement, son hagiographie. Sur la longue durée, la geste et l'œuvre de Mašrab sont replacées dans le sillage de la malāmatiyya, un courant gnostique médiéval apparu au Khorasan (est de l'Iran, ouest de l'Afghanistan, sud de l'Asie Centrale actuels) dès le III ${ }^{\mathrm{e}}$ siècle de l'hégire et qui exhortait à agir de façon blâmable pour protéger sa foi en cachant aux autres et à soi-même sa dévotion sous les apparences de la plus grande négligence (voir sur ce courant, en français, le très beau recueil de Sūlamī, poète malāmī du $\mathrm{x}^{\mathrm{e}}$ siècle : La lucidité implacable. Épittres des hommes du blâme, traduit de l'arabe et présenté par Roger Deladrière, Paris, Arléa, 1999). Papas rappelle (p.41) que si malāmatī et qalandar ne s'identifient pas, les seconds ont souvent emprunté aux premiers. Les chapitres consacrés à Mašrab fournissent nombre d'éléments d'histoire sur la radicalisation de la Qalandariyya centrasiatique, montrant notamment comment la littérature hagiographique fait avaliser cette évolution par le grand saint patron d'une culture de la marginalité, par exemple lorsque l'auteur oppose des récits de conversion à l'islam des chamanistes kirghizes, d'une part, et de l'autre un Mašrab aux allures de chamane confronté à des Kirghizes affectés du fanatisme des convertis : quelles que soient les circonstances, le qalandar se fait le défenseur des condamnés, des marginaux et c'est cette marginalité même qui fait de son œuvre personnelle comme de sa tradition posthume - presque toujours indiscernables l'une de l'autre - un révélateur de nombreux enjeux sociaux et culturels d'une époque de transition.

Le deuxième derviche errant $\mathrm{du}$ recueil, le gnostique originaire de Yarkand Muhammad-Ṣiddīq Dalīlī (1676-1753), quasi-inconnu de l'Occident, est abordé ici à travers deux copies préservées de son Récit de voyage (Safarnāma) en vers. Faisant partir son récit biographique du choix délibéré de la folie mystique (junūn) et de l'intensité du voyage - cheminement spirituel qui a pour mode l'abandon (tawakkul) de soi à Dieu l'auteur souligne aussi, d'emblée, la différence entre Dalīili et son prédécesseur Mašrab car, contrairement à ce dernier et à son désir de distinction par la conduite blâmable, Dalîlì cherche à se fondre dans les foules pieuses et à expliquer, à travers son itinéraire propre, la vie religieuse de tous (p.143). Une volonté de conciliation que l'auteur retrouve dans l'expression par Dalīlï d'une recherche de rapprochement, en plein XVIII siècle, c'est-à-dire à une époque tardive dans l'histoire de ces deux voies gnostiques (puisque leurs origines remontent au moins au XII ${ }^{\mathrm{e}}$ ) entre la Qalandariyya, réputée pour 
ses pratiques spectaculaires et ses postures hétérodoxes - « De grâce agrée mon oraison / Quelle que fût ma déraison ", invoque Dalīlī -, et la Naqšbandiyya qui s'ingénie à passer, elle, pour un parangon de sobriété, une championne du légalisme. L'évocation du culte des imams (sinon de l'imamisme pur et simple) chez Dalīlì permet aussi d'importants développements sur l'absence d'hostilité des qalandar à l'encontre du culte chiite des Abdals de Kachgharie (ces descendants des chiites de Koufa, en Mésopotamie méridionale, alors au cœur du Califat, réfugiés en Asie Centrale dès le $\mathrm{II}^{\mathrm{e}}$ siècle de l'Hégire, viII ${ }^{e}$ siècle de l'ère commune), et l'écho que reçoivent dans la tradition qalandarī, jusqu'à une date fort tardive, les imams du chiisme, vénérés comme héros et martyrs de l'islamisation de l'Asie Centrale. Mais l'essentiel chez Dalīlī est sa pratique du voyage gnostique, qui l'emmène jusqu'en Inde en quête de l'âme du maître disparu, devenu mort intercesseur. L'auteur montre bien comment, dans le Safarnāma, l'on retrouve un modèle d'errance cyclique très inspiré des visions du soufisme classique - depuis le cercle (hialqa) des disciples d'un maître jusqu'à la circumambulation (țawāf) rituelle aux lieux sacrés. D'un point de vue d'histoire sociale, le récit de Dalīlī semble confirmer (nulle autre source que le Safarnāma n'étant hélas convoquée) que la Qalandariyya est devenue à son époque une pratique courante: l'auteur rappelle que le XVIII ${ }^{\text {e }}$ siècle est, dans toute l'Asie Centrale, de part et d'autre des Tian-Shan, une époque où "le fou de Dieu, l'homme en haillons et aux cheveux emmêlés, se trouve dans chaque lieu de pèlerinage (mazār), dans les cimetières, les refuges, les hôtelleries de derviches " (p. 160-161). Dans les bazars aussi, où le qalandar côtoie deux protagonistes de la sociabilité gnostique et de la tradition hagiographique développées de siècle en siècle autour des saints musulmans gyrovagues : le maddāh et le qawwāl, panégyristes sacrés organisés en confréries, en groupes de statut aux fortes dimensions ethniques, faisant l'objet eux-mêmes d'une ségrégation forte (il suffit de mentionner la place des qawwāls d'origine jāt et de langue pachto venus de Qandahar sur les marchés de l'actuel Tadjikistan méridional jusqu'au début de l'époque soviétique.) Maddāḥ et qawwāl, malheureusement, sont absents du présent ouvrage, en dépit de l'importante littérature dont ils ont fait l'objet ces dernières décennies, tant de la part des historiens que des anthropologues de l'Afghanistan et de l'Asie Centrale.

5 Contemporain du précédent, le troisième et dernier héros du livre, 'Abd-Allah Nidāyī Kāšgarī (1688-1760) est peut-être celui dont la biographie est la mieux documentée. Il est vrai que son établissement sur les rives du Bosphore, à la fin de sa vie, et sa renonciation à l'errance et à la privation en faveur d'une vie sociale plus conventionnelle ont plaidé en faveur de l'éclosion à son sujet de toute une tradition biographique, suivie plus récemment d'une abondante production universitaire. L'auteur tente ici de réconcilier des points de vues parfois divergents en interprétant l'œuvre de Nidāyī comme «le terme ultime, parvenu à maturité, d'une résurgence du mode de vie qalandari parmi les milieux soufis d'Asie Centrale». Rien de moins. Issu comme ses deux prédécesseurs d'un milieu privilégié, le jeune 'Abd-Allah commence par être gratifié d'une éducation très classique jusqu'à sa " capture " à l'âge de dix-sept ans par un maître derviche errant, qui le met sur la voie du voyage au long cours. Chez Nidāyī comme chez ses prédécesseurs, le voyage continue d'être doublement sensible et spirituel: le soufi naqšbandì se doit de connaître les épreuves de la route et de l'indigence. Un chapitre entier de l'ouvrage est d'ailleurs consacré à la pauvreté chez Nidāyī, notamment à travers l'analogie entre pauvreté et anéantissement ( $\left.f a n \bar{a}^{\prime}\right)$, un thème déjà développé au XIII ${ }^{\mathrm{e}}$ siècle chez l'archi-classique Mawlānā Jalāl al-Dīn. Le récit de Nidāyī permet aussi de mettre au jour l'existence de tout un réseau de derviches 
vagabonds, englobant les voies naqšbandī d'une part, qādirī et qalandarì de l'autre, étendu dans l'espace de la périphérie de Boukhara aux profondeurs du Deccan (autre domaine d'interaction par excellence des traditions soufie et imamite). Il n'empêche : dans la lignée aussi de ses prédécesseurs, Nidāyī s'emploie à incarner un essai de synthèse entre Qalandariyya et Naqšbandiyya. Dans ses vers, pas d'errance proprement dite, le voyage n'ayant qu'une seule destination possible et nul égarement n'étant vraiment permis : « la folie est une discipline » (p. 243), résume efficacement l'auteur, et Nidāyĩ s'emploie à écarter tout soupçon de déviance. Ne trouve-t-on pas chez lui un exposé des onze règles de la Naqšbandiyya, présentée comme voie du diikr silencieux ( $h$ iu $\left.f_{i}\right)$ ? Du point de vue social, les observations du voyageur à Kachgar, au pied du mausolée d'Āfāq Hुwāja, tendent à montrer que l'aura de la dynastie naqšbandī continue d'attirer à elle les foules pieuses, et dans le sillage de Mašrab et de Dalîlī le poète invoque la pérennité d'une réputation du lieu saint. Son originalité réside-t-elle donc, outre les épisodes de sa biographie conclue à Istanbul, dans la résolution par Nidāyī de la tension du gnostique entre débordement amoureux et respect de la norme? L'installation à Istanbul s'est accompagnée de la renonciation du qalandar à la pauvreté et au célibat : un renoncement au renoncement (tark-i tark), comme chez Mawlānā Jalāl al-Dīn? suggère malicieusement l'auteur. L'interprétation que propose in fine l'ouvrage est celle du sentiment d'un monde finissant, qu'aurait partagé le qalandar avec l'ensemble de la société centrasiatique.

L'auteur doit être félicité pour proposer ici des éclairages si différents de l'histoire de l'islam centrasiatique et de ses multiples connections. Après un vaste panorama historique sur les interactions entre soufisme et politique au Xinjiang et un plus truculent récit de voyage chez les Salars du Qinghai, voici un ouvrage qui fait intelligemment alterner commentaire biographique et longs passages en vers traduits, dans un esprit qui n'est pas très éloigné de celui des "répertoires" (tadiākir) poétiques classiques arabes, persans et turciques, mais disposant de tout l'appareillage de la critique textuelle et historique la plus actuelle. L'auteur a aussi intégré l'apport des études et travaux d'érudition des chercheurs ouïghours et ouzbeks. Son exploitation originale et novatrice de la littérature hagiographique lui permet de mettre au jour "cette part de fait qui n'intéresse pas les chroniqueurs", selon l'intéressant postulat que c'est dans les vicissitudes banales que se révèle au mieux un certain état de la société, et qu'il n'y a peut-être pas de meilleur témoin de cette dernière qu'un poète en marge (p. 201-203). À propos des ressources heuristiques de l'hagiographie, l'auteur précise encore que ce n'est pas la cohérence, d'ailleurs improbable, du récit qui importe à l'historien, mais sa pertinence historique et en particulier la personnalité de ses acteurs (p. 233). De ce point de vue, l'ouvrage remplit toutes ses promesses, grâce à l'attention de son auteur pour le mélange des éléments concrets et de la vision gnostique dans nombre de descriptions laissées par les derviches qalandar (dans le Récit de voyage de Dalīlī tout particulièrement). Une textologie alimentée par une connaissance intime de la tradition littéraire soufie d'Asie Centrale et des travaux d'historiens comme Devin DeWeese lui permet d'identifier maints leitmotivs de l'hagiographie musulmane, parmi lesquels la conversion du prince païen - comme celle par Mašrab du khan bouddhiste Qūntajī. Autre lieu commun de la tradition littéraire persane et turque: la peur du Mongol - le saint vagabond « recueillant les peurs de ses coreligionnaires et mettant en vers le rêve d'un salut » (p. 72). 
7 L'ouvrage est également riche de nombreux éléments sur les aspects les plus divers de la vie interne des voies gnostiques musulmanes d'Asie Centrale, dont Papas s'est fait un historien majeur. Vient d'abord l'observation, récurrente, de l'indissociabilité des deux principales voies qui se développent dans la région au cours des siècles: la sobre Naqšbandiyya et la plus débridée Qalandariyya, les deux éléments d'une alternative capitale entre la route et le trône. De ce point de vue, les figures de Mašrab, de Dalīili et de Nidāyĩ sont ici opposées à celles des maîtres soufis régnants de Kachgarie et d'alentour, Hुwāja Ishāa et autres Āfāq Hुwāja dont l'auteur avait traité plus en détail dans son Soufisme et politique. Lecteur de Michel de Certeau, il insiste sur le caractère indistinctement spirituel et physique de la pérégrination qu'anime la quête spirituelle (p. 150 sq.). Aspect central de la voie qalandarī: les sociabilités en voyage, le derviche errant trouvant partout sur sa route une société d'amis de Dieu rassemblés autour de lieux de mémoire, les cimetières en particulier, qui contribuent à faire que la terre n'est plus orpheline car pleine des maîtres et des saints. À noter: de très nombreuses notations sur les routes de pèlerinage, en particulier celles de l'Iran safavide chiite, qui ne constitue nullement un obstacle et où Hamid Algar avait noté une importante présence naqšbandī - encore très forte sous la République islamique dans les marches orientales et occidentales du pays, comme l'ont noté de plus récents voyageurs. Plus importante peut-être, cependant, est ici la restitution d'une constante sociologique, que l'auteur qualifie de double hésitation du qalandar face à la communauté, et de la société face au fou, les uns comme les autres restant dans leur ambiguïté : Mašrab, qui tantôt respecte tantôt injurie les puissants, n'a-t-il pas été fou de Dieu au même titre que fou du roi, tandis que les khans et autres étaient tour à tour disciples, mécènes et bourreaux (une alternance de rôles qu'avait presqu'idéalement restituée le dramaturge Marc Weil dans la mise en scène de son propre Mašrab, où chacun des acteurs jouait alternativement chaque personnage).

Parmi les multiples aspects peu soulignés par l'historiographie actuelle du soufisme centrasiatique, mentionnons également la territorialisation de l'autorité d'un saint fondateur tel Satūq Bugrāa Hูān, dont l'aura spirituelle et la protection magique (baraka) couvrent un territoire (walāya) et qui possède sa province propre (wilāya), une composante essentielle de l'autorité spirituelle en Asie Centrale pré-moderne mais aussi moderne et contemporaine. L'ouvrage s'achève sur une interrogation: et si les trois figures évoquées à travers leurs œuvres propres comme à travers les traditions hagiographiques qu'elles ont inspirées n'étaient, en fait, pas autre chose que les créatures d'un désir populaire, «le vœu d'un contre-modèle, réponse au temps d'une société en fuite d'elle-même » (p. 266) ? L'historien de l'art Yves Porter avait évoqué les splendeurs des madrasas monumentales aux délicats décors peints de la même période, à Boukhara en particulier, comme procédant d'une volonté des dynasties régnantes de dissimuler une paupérisation croissante. Si l'on peut déplorer que le $\mathrm{xVIII}^{\mathrm{e}}$ siècle centrasiatique affleure très peu dans le récit qui nous est ici proposé, les questionnements soulevés par les sources particulières mises au jour touchent aux aspects les plus profonds d'une période historique de mutation, dont la résonnance se faisait encore sentir très récemment - les derniers témoignages sur la présence de la malāmatiyya dans les hautes vallées d'Asie Centrale méridionale remontent à la période Khrouchtchev (selon des témoignages oraux recueillis par l'auteur de ce compte rendu dans la moyenne vallée de Zerafchan, sur le territoire de l'actuel Tadjikistan, pendant l'été 2006). 
Certes, la traduction et le commentaire des textes et passages en vers de Mašrab, Dalīīi et Nidāyī tendent à éluder les aspects formels de cette poésie au profit du contenu, évacuant la dimension de virtuosité. Difficile au lecteur d'apprécier si ces poètes et leurs continuateurs apocryphes furent seulement des poètes passables. Impossible aussi d'établir un lien entre ces textes et la poésie de cour produite à la même époque. Cependant l'auteur évoque très bien, dans sa conclusion en particulier, l'érotisme gnostique teinté de provocation et la culture de l'anti-courtoisie persane qui s'étale dans les vers de nos derviches, notamment par le recours à un abondant vocabulaire argotique détourné - l'hôtellerie soufie devenant "lupanar» (lūlihhiāna ou, plus classiquement, hoarābāt), le contemplateur se trouvant réduit à la qualité de « mateur » (nazarbāaz), tandis que dans un ultime élan d'autodérision la société derviche tout entière se mue en "racaille " (qallăš). À signaler peut-être sur le plan purement formel, de menues erreurs de transcription, étonnantes compte tenu des contraintes que le mètre (wazn) de la prosodie arabo-persane fait peser sur l'alternance de voyelles "longues» et "brèves" (par exemple Rustām au lieu de Rustam); quelques approximations orthographiques aussi : les Kirghizs (une grosse faute d'orthographe en français) au lieu des Kirghiz ou Kirghizes; enfin, pourquoi Tashkent au lieu de Tachkent? Vétilles: le principal regret du lecteur un peu versé en histoire centrasiatique restera sans doute le silence du livre sur l'identité des protagonistes du processus hagiographique, maddāh-s et qawwāl-s en particulier, auteurs essentiels, autant que nos trois personnages, des biographies déroulées sous nos yeux. Rien ici qui permette d'en restituer les traits sociaux et culturels alors que c'est d'eux peut-être qu'il s'agissait ici de dresser le portrait, plus même que des figures en partie mythiques auxquelles leurs traditions écrites et orales ne cessent de renvoyer. Malgré ces menues réserves, l'ouvrage que propose Alexandre Papas est une lecture centrale, et tout à fait captivante, pour toute étude du processus hagiographique en général et en islam centrasiatique en particulier aux époques aussi bien pré-modernes et, en certains lieux, jusqu'au beau milieu du $x^{e}$ siècle. Raison de plus, peut-être, pour déplorer l'absence apparente d'intérêt de l'auteur pour l'époque contemporaine, en dépit des indéniables difficultés que comporte, au Turkestan chinois en particulier, l'étude de cette dernière. 